\title{
MICOBIOTA ENDÓFITA DEL GÉNERO MAXILLARIA RUIZ \& PAV. (ORCHIDACEAE) EN DOS RESERVAS NATURALES DEL DEPARTAMENTO DEL QUINDÍO
}

\author{
ENDOPHYTIC MYCOBIOTA OF GENUS MAXILLARIA RUIZ \& PAV. \\ (ORCHIDACEAE) IN TWO NATURE RESERVES OF QUINDÍO
}

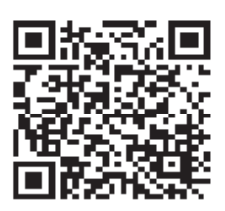

Johan Stiben Rodríguez Melo ${ }^{1}$, Fabiana María Lora Suarez ${ }^{2}$

${ }^{1 .}$ Estudiante de Licenciatura en Biología y Educación Ambiental - Universidad del Quindío.

2. Docente programa de Biología - Universidad del Quindío

Recibido: 6 Diciembre de 2015

Aceptado: 2 Febrero de 2016

*Correspondencia del autor: Johan Stiben Rodríguez Melo. E-mail: jsrodriguezm_1@uqvirtual.edu.co

\begin{abstract}
RESUMEN
Anteriormente las orquídeas eran consideradas como generalistas en sus interacciones con endófitos; tras el avance en el estudio de su fisiología se dio por sentado de que existe cierta variación en sus niveles de especificidad, incluso dentro de especies relacionadas filogenéticamente. Con el propósito de describir la micobiota endófita asociada al género Maxillaria en dos reservas naturales del departamento del Quindío. Se realizó un aislamiento y caracterización taxonómica de endófitos a partir de 576 fragmentos de tejido radicular provenientes de orquídeas pertenecientes al género Maxillaria. Se obtuvieron 268 asilados clasificados en 4 géneros de hongos endófitos; caracterizados macroscópica y microscópicamente; los géneros asilados con mayor frecuencia fueron los correspondientes al género-forma Rhizoctonia, representado por los géneros Tullasnela y Ceratobasidium. Se observaron diferencias en el número de plantas colonizadas por especie vegetal y por zona de muestreo. En conclusión, a pesar de ser un trabajo basado en caracteres morfotaxonómicos, se registran morfotipos del género forma-Rhizoctonia presentes en las zonas estudiadas, además este trabajo es la primera contribución Colombiana que documenta de manera sistemática algunos aspectos de la relación hongo orquídea en el departamento del Quindío y en el género Maxillaria.
\end{abstract}

Palabras claves: Interacciones, especificidad, aislados, colonización, morfotaxonómicos.

\begin{abstract}
Previously orchids were considered generalists in their interactions with endophytes; after the breakthrough in the study of physiology it was assumed that there is some variation in their levels of specificity, even within phylogenetically related species. In order to describe the endophytic mycobiota associated with the genus Maxillaria in two nature reserves in Quindio. Isolation and taxonomic characterization of endophytes was carried out from 576 fragments of root tissue from orchids of the genus Maxillaria. 268 inmates classified into 4 genres of endophytes were obtained; characterized macroscopically and microscopically; asylees genres most frequently were those relating to form genus Rhizoctonia, represented by genus Ceratobasidium and Tullasnela. Differences in the number of plant species colonized by sampling area and plants were observed. In conclusion, despite being a work based on morphotaxonomic characters, morphs of form genus Rhizoctonia are recorded in the study areas, in addition this work is the first Colombian contribution to systematically documenting some aspects of the relationship fungus-orchid from Quindío and genus Maxillaria.
\end{abstract}

Keywords: Interaction, specificity, isolated, colonization, morphotaxonomic. 


\section{INTRODUCCIÓN}

Colombia cuenta con cerca de 4.200 especies de orquídeas silvestres, agrupadas en 232 géneros (1). Sin embargo, en las últimas décadas se ha registrado una disminución alarmante de sus poblaciones en el país, debido al tráfico y comercialización ilegal de especies atractivas, además de otras actividades humanas como la minería y la ampliación de fronteras agrícolas y ganaderas (2). Alexander (3) plantea que estas últimas han provocado la modificación drástica de ecosistemas naturales, afectando la distribución y abundancia de gran número de especies vegetales, incluyendo las orquídeas.

La literatura sobre hongos endófitos y hongos formadores de micorrizas en orquídeas está estrechamente relacionada y a veces es imposible discutir estas asociaciones por separado. El termino endófito se refiere a los microorganismos que crecen dentro de los tejidos vegetales sin causar síntomas de enfermedad pero sin asumir o excluir la posibilidad de beneficio para ambas partes (4-6). Por el contrario, el concepto de micorriza se enfoca en la parte funcional y describe una relación comúnmente mutualista (7-9). Estudios recientes como los de Bayman y Otero (4) y Tan et al., (10) resaltan el papel que cumplen los endófitos en la protección de la planta frente a ataques de patógenos, ya sea por la síntesis de metabolitos secundarios o por el mejoramiento en la nutrición a través de la disponibilidad de los nutrientes (11). A pesar de esto, estudios como los de Bayman et al., (12) muestran la falta de profundización en el conocimiento de las implicaciones que tienen dichas asociaciones y ponen en discusión la necesidad de conocer más a fondo temas como la importancia de los endófitos en la fisiología de las plantas hospedera y su verdadera significancia funcional para lo cual, en primera instancia, se hace necesaria una descripción de los endófitos que comúnmente se asocian a las diferentes especies de orquídeas.

El género Maxillaria además de la vistosidad de sus flores y complejas formas, se caracteriza por presentar una amplia distribución a lo largo y ancho del territorio quindiano $(1,13-17)$, por lo que podrían ser parte fundamental de los ecosistemas de la región. La implementación de herramientas como el uso adecuado de microorganismos, específicamente organismos endófitos asociados a las raíces terrestres y hongos endófitos formadores de micorriza en orquídeas, sin dis- criminar importancia económica u ornamental, brinda la posibilidad de generar estrategias de conservación enfocadas en la incursión del uso de herramientas amigables con el ambiente (18); otorgando no solo el reconocimiento a países como Colombia por el uso de nuevas herramientas en pro de la conservación, sino también la ampliación en el conocimiento de relaciones ecológicas tan complejas como lo es el caso de las orquídeas y sus endófitos.

Con respecto al aislamiento y descripción de hongos endófitos presentes en orquídeas epífitas neotropicales, poco se sabe al respecto. Girlanda et al., (19) en aislados obtenidos de pelotones en orquídeas de Brasil reveló la presencia de los anamorfos Epulorhiza repens, Epulorhiza epiphytica, Epulorhiza spp. у Ceratorhiza spp. En el caso particular de Colombia y especialmente en el departamento del Quindío, pocos han sido los trabajos que han documentado los endófitos asociados a orquídeas. Otero y Bayman (20), dan un listado preliminar de algunos géneros-forma, aislados de orquídeas del género Vanilla en Colombia. Sin embargo, sobre los hongos asociados a otras especies de orquídeas epifitas y terrestres no existe ningún trabajo (21) y el presente constituye el primer registro para especies de orquídeas en el departamento del Quindío.

Considerando la riqueza de especies de orquídeas en nuestro país y la importancia ecológica de los hongos endófitos, el presente trabajo se realizó con el objetivo de describir la micobiota endófita asociada al género Maxillaria en dos reservas naturales del departamento del Quindío, Colombia.

\section{MATERIALES Y MÉTODOS.}

Área de estudio: La reserva natural La montaña del Ocaso está localizada en la vereda El Laurel, al sur del municipio de Quimbaya y noroccidente del municipio de Montenegro, en el departamento del Quindío

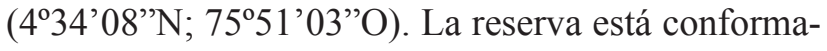
da por un relicto selvático intervenido de 106 hectáreas, que presenta un rango altitudinal entre los 9001000 msnm en la confluencia de los ríos La Vieja y El Roble (15).

La reserva natural La Sonadora, finca La Merced (4²6'17,6”N; 75³7'21,3'W) está ubicada en el municipio de Calarcá (Quindío), sobre la vertiente occidental de la Cordillera Central, entre los 3.000 a 3.150 
msnm. Hace parte de las 2.000 ha que el Comité de Cafeteros del Quindío destinó para la conservación y restauración ecológica de la cuenca alta de los ríos Santo Domingo y Río Verde.

\section{Recolección del material biológico}

Se recolectaron seis individuos por especie registrada en cada una de las reservas, $\left(\mathrm{RNMO}^{1}\right.$ : Maxillaria acuminata $^{2}$, Maxillaria guareimensis y Maxillaria equitans; RNS ${ }^{3}$ : Maxillaria procurrens, Maxillaria alticola,). Muestreándose un total de 36 ejemplares en ambas zonas, los cuales fueron empacados en bolsas individuales y transportados hasta el laboratorio de investigaciones del grupo BIOEDUQ donde se procedió a retirar, de cada ejemplar, las raíces que se observaban en mejor estado, para luego ser almacenadas en bolsas estériles termosellables a $4^{\circ} \mathrm{C}$.

\section{Aislamiento de hongos endófitos.}

La determinación del medio de cultivo a usar se basó principalmente en las características biológicas y fisiológicas de los hongos microscópicos. El medio de cultivo PDA enriquecido con ácido láctico tiene la infusión de papa como fuente de almidones, los cuales, en conjunto con la dextrosa son la base para el crecimiento de hongos y levaduras. El bajo $\mathrm{pH}$ (3.5) evita el crecimiento de las bacterias. Es común utilizarlo en estudios de identificación de hongos debido a que mantiene activa la patogenicidad y capacidad de producir propágulos de reproducción de los mismos.

Por cada planta se analizaron cuatro tejidos radiculares sanos, los de mayor edad (Mayor tamaño y espesor). Cada fragmento de tejido fue desinfectado superficialmente de forma individual en soluciones estériles, de acuerdo con el protocolo establecido por Otero et al., (22) con modificaciones. Los fragmentos se sumergieron en agua destilada estéril durante 30 segundos tres veces, y posteriormente, en solución de etanol al 70\% durante 60 segundos, seguido por lavado en hipoclorito de sodio al $1 \%$ durante 5 minutos y etanol al $70 \%$ por 30 segundos. Finalmente, los fragmentos se enjuagaron con agua destilada estéril para eliminar el exceso de hipoclorito y etanol y se secaron con papel absorbente estéril. Cada tejido de raíz desinfectado se cortó en 4 fragmentos de $0,25 \mathrm{~mm}$ los

\footnotetext{
${ }^{1}$.Reserva natural La Montaña del Ocaso

${ }^{2}$.Especie registrada en ambas reservas

${ }^{3}$.Reserva natural La Sonadora
}

cuales fueron colocados en secciones claramente definidas (A, B, C, D) en cajas Petri con medio de cultivo agar papa dextrosa (PDA, Merck), enriquecido con ácido láctico $(500 \mathrm{uL} / 11 \mathrm{t})$.

Los cultivos fueron incubados durante 15 días a temperatura ambiente registrando cada $24 \mathrm{~h}$ la presencia o ausencia de colonias en el medio. Como control del proceso de desinfección se sembraron en PDA cinco alícuotas de $1 \mathrm{ml}$ del agua destilada del enjuague final, ya que si la eliminación de la microbiota epífita fue efectiva esta solución debe estar estéril. Los controles también fueron incubados por 15 días.

\section{Identificación de los hongos endófitos.}

Para la caracterización taxonómica de los hongos aislados a partir de raíces de orquídeas, siguiendo el protocolo establecido por Otero et al., (22) con modificaciones, se realizaron cultivos con tres repeticiones de los hongos mediante rayado vertical, para obtener un cultivo puro y homogéneo. Los hongos purificados fueron almacenados en un sitio libre de contaminación a temperatura ambiente por un lapso de 15 días, hasta su maduración y determinación de características morfológicas.

Mediante observación directa se realizó la comparación de características macroscópicas y microscópicas con estructuras morfológicas y con las claves taxonómicas de Barnett y Hunter (23) para géneros de hongos microscópicos.

Para la identificación macroscópica de los hongos aislados se realizó la observación de características como: textura, coloración del micelio, presencia de esclerocios y morfología.

La observación microscópica de los hongos aislados se llevó a cabo mediante el montaje al microscopio óptico compuesto de fragmentos del cultivo, usando la técnica de cinta adhesiva, realizando comparaciones de características como: Hifas (alargadas o anchas, ramificadas, con septos); estructuras de almacenamiento de esporas (esporangios o conidios); ausencia o presencia de esporas.

\section{Análisis de los datos}

La incidencia de hongos endófitos fue comparada a nivel de especie y zona de muestreo. 
La frecuencia de colonización (\% FC) de cada género fue calculada como la relación entre el número de fragmentos positivos para el género y el número total de fragmentos muestreados, expresado como un porcentaje:

$\% F C=\frac{\# \text { de fragmentos positivos para el généro füngico }}{4 \text { fragmentos verdaderos muestreados por tejido/planta }} * 100$

La abundancia relativa de hongos se definió como el número de aislados por género dividido en el número total de aislados por especie de orquídea.

La incidencia de hongos fue analizada a nivel de género y especie de orquídea en cada zona por separado, ésta se realizó mediante un análisis de componentes principales utilizando el software InfoStat, donde fue determinada la distribución de los géneros de endófitos en relación a las especies de orquídeas y a la zona de estudio. Tomando como referencia los datos correspondientes a la abundancia relativa de cada género de hongo y frecuencia de colonización que presentó en cada especie de orquídea e interpretando en la gráfica la distancia entre los puntos correspondientes a géneros de endófitos y especies de orquídeas, considerando la cercanía entre puntos como mayor incidencia del género de endófito sobre la especie de orquídea.

\section{RESULTADOS Y DISCUSIÓN.}

Identificación de los hongos endófitos: Se obtuvieron 576 fragmentos radiculares a partir de los 36 ejemplares colectados en ambas reservas. El crecimiento de hongos endófitos en los fragmentos fue evidenciado después de ocho días de incubación.

La totalidad de fragmentos positivos fue del $46,5 \%$, a partir de los cuales se obtuvieron 268 aislados fúngicos clasificados en 4 géneros (Figura 1) dentro de los cuales se reportan algunos que comúnmente causan enfermedades en otras plantas como lo son Ceratobasidium, Tulasnella y Colletotrichum.

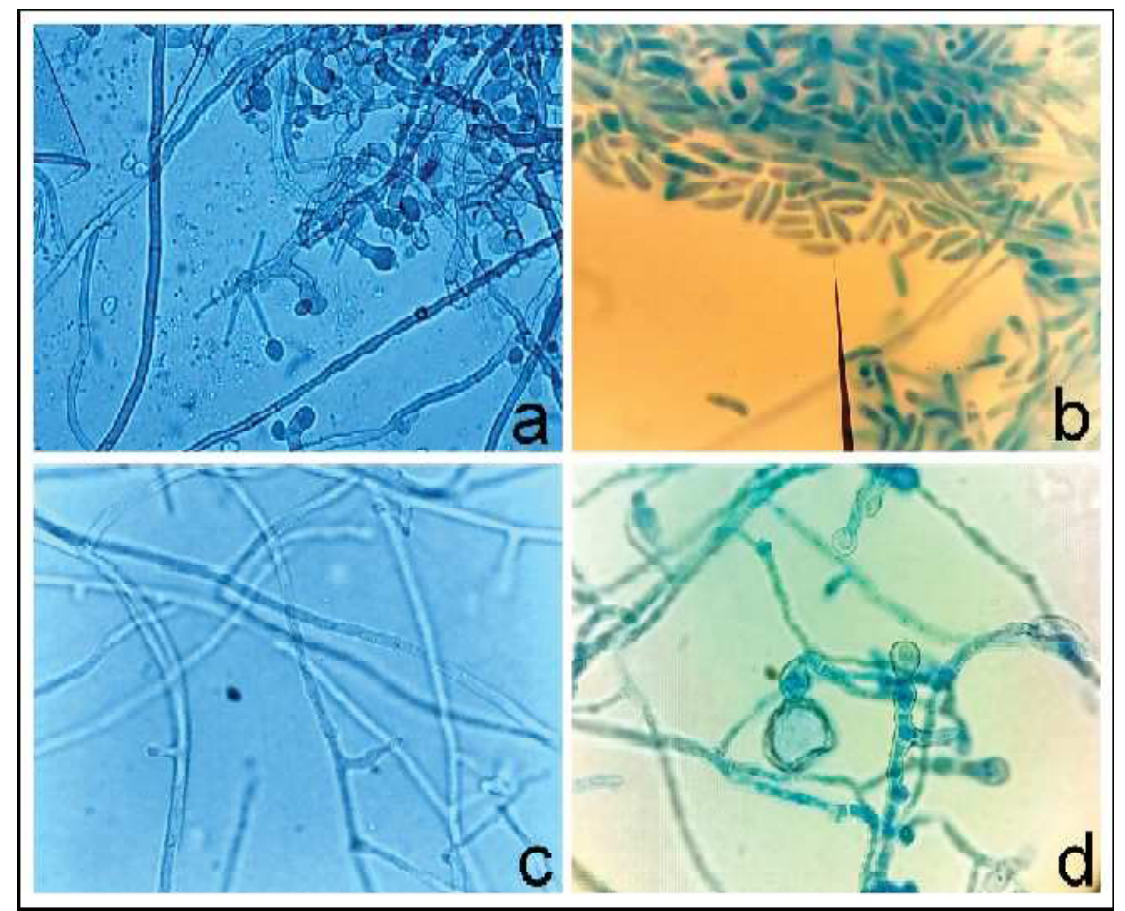

Figura 1. Endófitos aislados. a. Ceratobasidium; b. Colletotrichum; c. Sclerotium; d. Tullasnela.

Según Newton et al., (24) las plantas interactúan con comunidades microbianas establecidas en la superficie y al interior de sus tejidos, constituyendo así un sistema complejo denominado microbioma que da lugar a interacciones positivas y negativas. Teniendo en cuenta dichas interacciones Porras- Alfaro y Bayman
(25) reportan que una característica de los microorganismos endófitos es la colonización asintomática del interior de los tejidos y su posterior establecimiento como mutualistas, comensalistas o en algunos casos patógenos o saprófitos, estos últimos causando sintomatologías variadas en las plantas. 


\section{Riqueza de la micobiota endófita}

Los géneros Ceratobasidium y Tulasnella fueron los endófitos que presentaron una mayor abundancia relativa $(>0,25)$ con respecto a los demás géneros de endófitos (Figura 2).

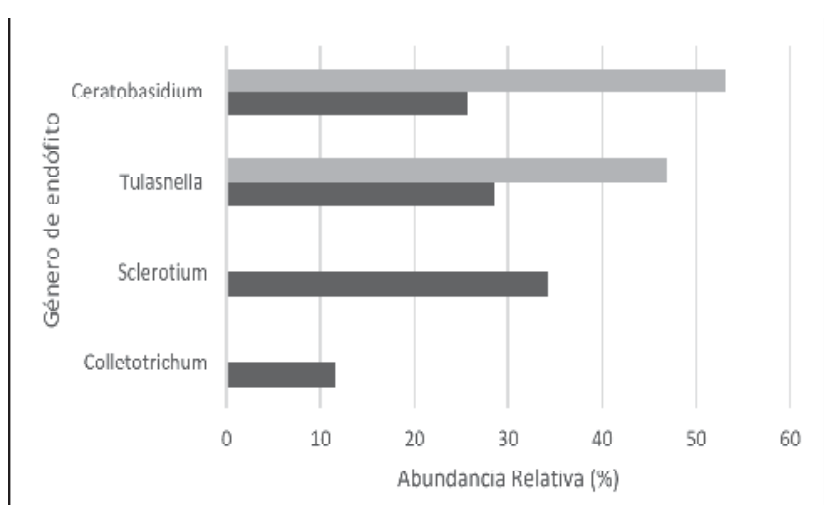

RNS $\square$ RNMO

Figura 2. Abundancia relativa de endófitos. RNS (Reserva natural La Sonadora); RNMO (Reserva natural La Montaña del Ocaso).

Las abundancias relativas registradas muestran una diferencia en los géneros presentes en ambas zonas, por lo que la altitud podría ser considerada como un factor limitante de la distribución y posible asociación de estos hongos con orquídeas, especialmente los géneros Sclerotium y Colletotrichum los cuales estuvieron restringidos solo a la zona de la Reserva Natural La Sonadora (Figura 2). Giusiano et al., (26) plantea que el desenvolvimiento de los hongos endófitos experimenta, asimismo, la influencia de los factores ecológicos, no sólo por ser ellos sensibles a dicha acción, sino también porque la receptividad de las plantas varía según cambian las condiciones del suelo, humedad, sequedad, altitud etc. probablemente la altitud a la cual se encuentran las orquídeas en la reserva natural La Montaña del Ocaso es un factor limitante para el establecimiento de algunos géneros de endófitos, ya sea por el cambio en la receptividad de las orquídeas o por presión ecológica.

El análisis de componentes principales agrupo las variables obtenidas de la abundancia relativa de los endófitos, la zona de muestreo y las especies de orquídeas (Figuras 3 y 4); explicando para el caso de la reserva natural la Montaña del Ocaso el $83,3 \%$ de la variabilidad de los datos (Figura 3), mientras que para la reserva natural La Sonadora el análisis explicó el $51,3 \%$ de la variabilidad de los datos (Figura 4).

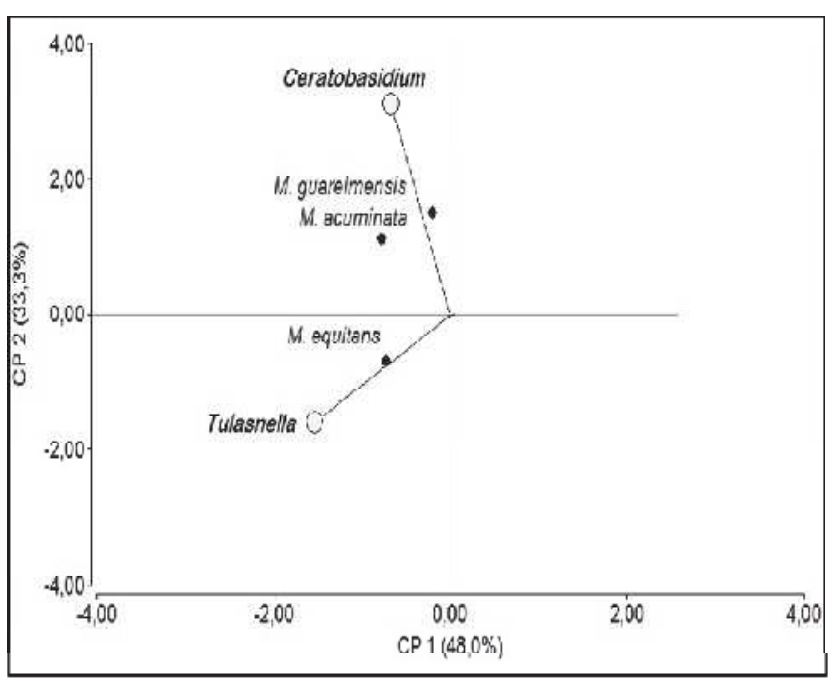

Figura 3. ACP géneros de endófitos-vs-especies de orquídeas en la reserva natural La Montaña del Ocaso.

Teniendo en cuenta el análisis de componentes principales para la reserva natural La Montaña del Ocaso (Figura 3), la incidencia de los géneros de endófitos aislados muestra que estos se encontraron distribuidos aleatoriamente, de tal manera que no se reportó preferencia o especificidad de endófitos por alguna especie en particular del género Maxillaria. En un estudio similar Ordoñez (21) menciona que en orquídeas no hay evidencia de hongos endófitos hospedero-específico. Los endófitos que fueron registrados tanto en este estudio como en otros, corresponden a géneros aislados en otras plantas y en casos específicos como Ceratobasidium y Tulasnella han sido relacionados con graves infecciones en otras plantas. Así mismo Bayman y Otero (4) en un estudio realizado con orquídeas terrestres y epifitas encontraron que las diferencias en la incidencia de géneros de endófitos pueden variar dependiendo del hábito, hábitat, forofito, entre otros aspectos relacionados con presiones ecológicas que limitan tanto la distribución del hongo como la incidencia del mismo en determinadas plantas.

El análisis de componentes principales para la reserva natural La Sonadora (Figura 4) la incidencia de endófitos en orquídeas muestra que tampoco se reporta preferencia o especificidad de endófitos por alguna especie de orquídea, excepto en $M$. procurrens que presentó aislados únicos correspondientes al género Sclerotium (Figura 4). 


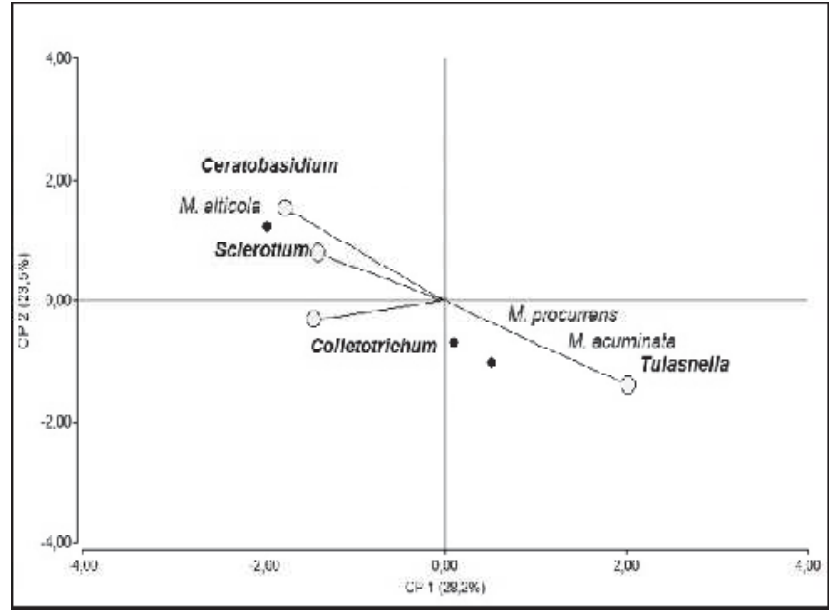

Figura 4. ACP géneros de endófitos-vs-especies de orquídeas en la reserva natural La Montaña del Ocaso.

Para Hadley y Williamson (27), Benzing (28) y Goh et al., (29) la ausencia de endófitos en algunas raíces aé $\neg$ reas, así como la incidencia discontinua o aislados fúngicos únicos, también observada en otras especies, refuerza la importancia del papel del substrato para el establecimiento del endófito. En las orquídeas terrestres la infección en las nuevas raíces ocurre rápidamente mediante propágalos existentes en el suelo o por raíces infectadas o muertas el año anterior (30); M. procurrens se caracteriza por presentar hábitos terrestres y encontrarse en grandes colonias con individuos que se regeneran constantemente, lo cual podría estar explicando la presencia de un solo género de hongo.

Los géneros Ceratobasidium y Tulasnella fueron aislados tanto en orquídeas terrestres como de orquídeas con hábito epifito, lo cual apoya la evidencia que las orquídeas epífitas están colonizadas por micorrizas de la misma manera que las orquídeas terrestres. Rivas, Warner y Bermúdez (31) reportaron que en la mayoría de especies epífitas la infección es necesaria para la germinación de las semillas, en su estudio utilizaron 12 especies tanto de orquídeas terrestres como epífitas; de la misma manera Suárez et al., (2) utilizaron 20 especies epífitas de orquídeas y ambos estudios concluyeron que la mayoría de los individuos epífitos utilizados en sus investigaciones presentaron una densa colonización por hongos, similar a la encontrada en el presente estudio en los individuos con hábitos terrestres. Ambos géneros de endófitos pertenecientes al género-forma Rhizoctonia; han sido reportados en Australia por Warcup y Talbot (32), Otero et al., (33), Escocia por Warcup y Talbot (32), Puerto Rico por Otero et al., (22, 33-36); Porras-Alfaro y Bayman, (25), y Colombia por Mosquera-Espinosa et al., (37). Este género-forma es un patógeno de distribución mundial, asociado a enfermedades en plantas del género Solanaceae y es ampliamente conocido por las pérdidas que ocasiona, especialmente en la mayoría de las plantas anuales y perennes incluyendo casi todos los cultivos hortícolas que se desarrollan dentro o sobre el suelo (38).

El género Sclerotium se encuentra fuertemente relacionado con enfermedades asociadas a especies del género Catleya. Rivera-Coto y Corrales-Moreira (39) lo clasifican como agente causal de pudriciones en la raíz de orquídeas del género Phalaenopsis y en la base de la planta en Spathoglottis. En el presente estudio los aislados de este género provenían de plantas completamente saludables, por lo que Sclerotium al ser aislado en el 96\% de las muestras radiculares podría estar cumpliendo un papel fundamental en el establecimiento de las orquídeas de la reserva natural La Sonadora.

Rasmussen y Whitham (40) argumentan que las fluctuaciones observadas en los endófitos, pueden estar influenciadas por eventos climáticos como la lluvia o la sequía. En éste caso según datos de la estación meteorológica La española (Quimbaya) los registros pluviométricos de antes y durante la colecta de ejemplares en la reserva natural La Montaña del Ocaso muestran una baja precipitación (Tabla 1), mientras que según datos de la estación meteorológica La Línea (Calarcá) en la reserva natural La Sonadora se registraron altas precipitaciones (Tabla 1) antes y durante la fase de muestreo de ejemplares, razón por la cual la diversidad de endófitos formadores de micorrizas en zonas geográficas o épocas del año con diferencias en variables altitudinales y climatológicas constituye un tema de enorme interés, el cual no ha sido estudiado. Rains et al., (41) ha reportado que en plantas epifitas distintas de las orquídeas, que se asocian a micorriza arbuscular y ericoide, la diversidad micorrízica está directamente influenciada por el contenido de humedad en el sustrato orgánico o por la humedad atmosférica, siendo de gran importancia la presencia de musgos en los forófitos y la fertilidad de los suelos. 
Tabla 1. Registro climatológico para ambas reservas. Fuente: Estaciones meteorológicas CRQ y Proyecto Túnel de la Línea.

\begin{tabular}{|c|c|c|c|c|c|c|}
\hline \multirow[b]{3}{*}{ Zona } & \multirow[b]{3}{*}{ Variable } & \multirow{2}{*}{\multicolumn{5}{|c|}{ Mes (2015) }} \\
\hline & & & & & & \\
\hline & & Enero & Febrero & Marzo & Abril & Mayo \\
\hline \multirow{3}{*}{ RNS* } & \multirow{3}{*}{$\begin{array}{l}\text { Temperatura }\left({ }^{\circ} \mathrm{C}\right) \\
\text { Humedad }(\%) \\
\text { Prec ip i ta ción } \\
(\mathrm{mm})\end{array}$} & 10,3 & 11,6 & 10,5 & 11,6 & 10,3 \\
\hline & & 85 & 86 & 87 & 86 & 86 \\
\hline & & 248,6 & 340,2 & 232,4 & 173,5 & 171,7 \\
\hline \multirow{3}{*}{ RNMO** } & \multirow{3}{*}{$\begin{array}{l}\text { Temperatura }\left({ }^{\circ} \mathrm{C}\right) \\
\text { Humedad }(\%) \\
\text { Precipitación } \\
(\mathrm{mm})\end{array}$} & 24,3 & 25,6 & 24,1 & 25,7 & 24,7 \\
\hline & & 70 & 72 & 72 & 72 & 71 \\
\hline & & 9,6 & 25,1 & 49,2 & 27,3 & 55,4 \\
\hline
\end{tabular}

En conclusión a pesar de ser un trabajo basado solo en caracteres morfotaxonómicos, existen morfotipos del género forma-Rhizoctonia presentes en los hábitats estudiados. Los caracteres morfotaxonómicos coinciden con dos de los géneros Teleomorficos frecuentemente aislados en orquídeas terrestres y epifitas: Ceratobasidium y Tulasnella. También es importante resaltar que más de un morfotipo estuvo presente en una misma sección de raíz, como ha sido reportado por otros autores. Además que, en condiciones donde dos o más especies crecen relativamente cerca una de otra, es posible que compartan los mimos simbiontes fúngicos, esto hace hincapié, que más que una especificidad por especie haya una afinidad según el sitio.

Finalmente se hace necesario aumentar los estudios para conocer más a fondo estas interacciones, la importancia del papel que cumple en la fisiología tanto de la planta hospedera como del endófito y su significancia funcional. Para posteriores investigaciones en endófitos de orquídeas se plantea la necesidad de: realizar una taxonomía filogenética de los microorganismos aislados, determinar los efectos fisiológicos y consecuencias de la eficiencia de estos sobre sus hospederos, así mismo, la interacción que se presenta con otros organismos endófitos asociados a las plantas. De esta forma, se podría determinar el potencial de estos hongos en el control de patógenos (fungicidas y bactericidas), resistencia al estrés, adquisición de nutrientes etc., haciendo más competitivos los cultivos.

\section{AGRADECIMIENTOS.}

Los autores agradecen al grupo BIOEDUQ y al almacén de reactivos y materiales de la universidad del Quindío por el apoyo logístico, durante la realización de la presente investigación. 


\section{BIBLIOGRAFÍA}

1. Sarmiento, J. (2007). La familia Orchidaceae en Colombia, Actualidades biológicas, 29 (1): 21-84.

2. Suárez, J. P., Weib, M., Abele, A., Garnica, S., Oberwinkler. (2006). Diverse tulasnelloid fungi form mycorrhizas with epiphytic orchids in an Andean cloud forest. Mycol Res, 110: 1257-1270.

3. Alexander, M. (1994). Introducción a la microbiología del suelo. AGT Editor, S.A. 491 p.

4. Bayman, P., y Otero, J. T. (2006). Microbial endophytes of orchid roots. En: Schulz B, Boyle C, Sieber T, editores. Microbial root endophytes. New York: Springer. p. 153-173.

5. Brundrett, M. C. (2006). Understanding the roles of multifuncional mycorrhizal and endophytic fungi. En: Schulz, B., Boyle, C. J., y Sieber, T. N. (eds.), Microbial root endophytes, 9: 281-298.

6. Stone, J. K., Bacon, C.W., y White, J. F. (2000). An overview of endophytics microbes: Endophytism defined. En: Bacon, C. D y White, J. F. (eds) Mycrobial Endophytes, 12: 3-29.

7. Smith, S.E., y Read, D.J. (1997). Mycorrhizal Symbiosis. Academic Press, London.

8. Rasmussen, H. N., y Whigham, D. F. (2002). Phenology of roots and mycorrhiza in orchid species differing in phototrophic strategy. New Phytol, 154: 797-807.

9. Cameron, D. D., Johnson, I., Leake, J. R., y Read, D. J. (2007). Mycorrhizal Acquisition of Inorganic Phosphorus by the Green-leaved Terrestrial Orchid Goodyera repens. Annals of Botany, 99: 831-834.

10. Tan, X. M., Chen, X. M., Wang, C. L., Jin, X. H., Cui, J. L., Chen, J., Guo, S. X., Zhao, L. F. (2012). Isolation and identification of endophytic fungi in roots of nine Holcoglossum plants (Orchidaceae) collected from Yunnan, Guangxi, and Hainan provinces of China. Current Microbiology, 64 (2): 140-147.

11. Schulz, B. y Boyle, C. (2006). What are endophytes? En: B. Schulz., Boyle, C. J., y Sieber, T. N. (eds.), Microbial root endophytes, 9: 1-13.

12. Bayman, P., Lebrón, L. L., Tremblay, R. L. y Longe, D. J. (1997). Variation in endophytic fungi from roots and leaves of Lepanthes (Orchidaceae). New Phytologist, 135: 143-149.

13. Viveros, P., Molina, J., y Vélez, C. (2001). Inventario de la familia Orchidaceae en la Selva "La Montaña del Ocaso", Departamento del Quindío. Monografias de la Flora Andina, 3(1), 59-118.

14. Borsic, I. (2003). Contribution to the epiphytic orchid of the nature reserve "La Montaña del Ocaso" Quindìo, Colombia.

15. Santa, N., García, T y Gómez, G. (2009). Estructura y composición de la comunidad de orquídeas en dos zonas de la Reserva Natural "La Montaña del Ocaso" Quimbaya-Quindío. rev. invest. univ. Quindio, (19): 122- 134.

16. López, A., Gómez, G., y Sepúlveda, M. (2009). La sonadora: Ecosistema estratégico para la biodiversidad. Armenia, Quindio: Universidad del Quindío.

17. Berrio, C. (2011). Flora orquideológica de un relicto boscoso de alta montaña del departamento del Quindio (Tesis de pregrado). Centro de Estudios e Investigaciones en Biodiversidad y Biotecnología CIBUQ, Universidad del Quindío, Armenia, Colombia.

18. Bermúdez, D., y Benzing, D. H. (1989). Fungi in neotropical epiphyte roots. BioSystems, 23: 65-73.

19. Girlanda, M., Selosse, M. A., Cafasso, D., Brilli, F., Delfine, S., Fabbian, R., Ghignone, S., Pinelli, P., Segreto, R., Loreto, F., Cozzolino, S., y Perotto, S. (2006). Inefficient photosynthesis in the Mediterranean orchid Limodorum abortivum is mirrored by specific association to ectomycorrhizal Russulaceae. Molecular Ecology, 15: 491-504.

20. Otero, J. y Bayman, P. (2009). Germinación simbiótica y asimbiótica en semillas de orquídeas epifitas. Acta Agronomica, 58(4), 270-276.

21. Ordoñez, N. F. (2012). Efecto de hongos endófitos de orquídeas del grupo Rhizoctonia y otros endófitos cultivables sobre el desarrollo de plantas de Vanilla planifolia J. (Tesis de grado) Universidad Nacional de Colombia Sede Medellín. Facultad de Ciencias Agropecuarias. Maestría en Bosques y Conservación Ambiental.

22. Otero J., Ackerman J. y Bayman P. (2002). Diversity and host specificity of endophytic Rhizoctonialike fungi from tropical orchids. American Journal of Botany, 89 (1), 1852-1858.

23. Barnett, H., y Hunter, B. (2003). Ilustrated Genera of Imperfect Fungi. Minessota: Prentice-Hall. 
24. Newton, A., Fitt, B., Atkins, S., Walters, D., y Daniell, T. (2010). Pathogenesis, parasitism and mutualism in the trophic space of microbe-plant interactions. Trends in Microbiology, 18 (8): 365-373.

25. Porras-Alfaro, A. y Bayman, P. (2011). Hidden fungi, emergent properties: endophytes and microbiomes. Annual Review of Phytopathology, 49: 291-315.

26. Giusiano, G., Piontelli L., Mangiaterra, E., y Sosa, M. (2002). Distribución altitudinal de hongos queratinofilos, epífitos y endófitos en suelos semiáridos del noreste argentino (Prov. de Jujuy, $23^{\circ} \mathrm{L}$. S y 66ㄴ. W). Biol. Micol. 17: 51-62.

27. Hadley, G. y Williamson B. (1972). Features of mycorrhizal infection in some Malayan orchids. New Phytol. 71: 1111-1118.

28. Benzing, D. H. (1982) Mycorrhizal infection of epiphytic orchids in southern Florida. Amer Orchid Soc Bull, 51: 618-622.

29. Goh, C. J., Sim, A. A., y Lim, G. (1992). Mycorrhizal associations in some tropical orchids. Lindleyana 7:13-17.

30. Bridge,W. C. (1979). The ecology of fungy. CRC, Boca Raton, Florida, p.187-190.

31. Rivas, R. M., Warmer, J., y Bermúdez, M. (1998). Presencia de micorrizas en orquídeas de un jardín botánico neotropical. Rev Biol Trop, 46: 211-216.

32. Warcup, J. H. y Talbot, P. (1967). Perfect estates of Rhizoctonias associated with orchids. New Phytologist, 66: 631-641.

33. Otero, J. T., Thrall, P. H., Clements, M., Burdon, J.J., y Miller, J.T. (2011). Codiversification of orchids (Pterostylidinae) and their associated mycorrhizal fungi. Australian Journal of Botany 59(5): 480-497.

34. Otero J., Ackerman J. y Bayman P. (2004). Differences in mycorrhizal preferences between two tropical orchids. Molecular Ecology, 13(1), 2393-2404.

35. Otero J., Ackerman J. y Bayman P. (2005). Individual variation in plant and fungus for mycorrhizal seed germination in an epiphytic orchid. Evolutionary Ecology, 19(1), 29-43.

36. Otero J. T., Flanagan, N. S., Herre, E. A., Ackerman J. D. y Bayman, P. (2007). Widespread mycorrhizal specificity correlates to mycorrhizal function in the neotropical, epiphytic orchid, Ionopsis utricularioides. American Journal of Botany, 94: 1944-1950.

37. Mosquera-Espinosa, A., Bayman, P., y Tupac, J. (2010). Ceratobasidium como hongo micorrízico de orquídeas en Colombia. Acta Agronomica, 59(3), 316 - 326.

38. Sharon, M., Kuninaga, S., Hyakumachi, M., y Sneh, B. (2006). The advancing identification and classification of Rhizoctonia spp. Using molecular and biotechnological methods compared with the classical anatomosis grouping. Mycoscience, 47: 299-316.

39. Rivera-Coto, G., y Corrales-Moreira, G. (2007). Problemas fitosanitarios que amenazan la conservación de las orquídeas en costa rica. Lankesteriana, 7(1-2): 347-352.

40. Rasmussen, H. N., y Whigham, D. F. (2002). Phenology of roots and mycorrhiza in orchid species differing in phototrophic strategy. New Phytol, 154: 797-807.

41. Rains, K.C., Nadkarni, N. M., y Bledsoe, C. S. (2003). Epiphytic and terrestrial mycorrhizas in a lower montane Costa Rican cloud forest. Mycorrhiza, 13: 257-264. 